\title{
Treatment of senile trigeminal postherpetic neuralgia
}

\author{
Hao Zhou ${ }^{1}$, Zhao Gao ${ }^{1}$, and Yanjun Sun ${ }^{1}$ \\ ${ }^{1}$ Southeast University Zhongda Hospital
}

October 31, 2021

\begin{abstract}
The treatment effect of trigeminal postherpetic neuralgia is often unsatisfactory. We describe a case of low temperature plasma technique combined with lateral injection of trigeminal ganglion in the treatment of senile trigeminal postherpetic neuralgia.

Treatment of senile trigeminalpostherpetic neuralgia

Zhou $\mathrm{Hao}^{1} \mid$ Gao Zhao $^{1}$ | Sun Yanjun ${ }^{1}$

${ }^{1}$ Depertment of Pain Management, Zhongda Hospital Southeast University, Nanjing, China
\end{abstract}

Correspondence

Sun Yanjun, Depertment of Pain Management, Zhongda Hospital Southeast University, 87 Dingjiaqiao, Nanjing, China.

Email: 517989363@qq.com

\section{Key Clinical Message}

Treatment of trigeminal postherpetic neuralgia is often only partially effective. We treated an elderly case of trigeminal postherpetic neuralgia successfully by low-temperature plasma technology combined with injection of trigeminal ganglion.

\section{Abstract}

The treatment effect of trigeminal postherpetic neuralgia is often unsatisfactory. We describe a case of low temperature plasma technique combined with lateral injection of trigeminal ganglion in the treatment of senile trigeminal postherpetic neuralgia.

\section{KEYWORDS}

trigeminal postherpetic neuralgia, low temperature plasma, injection of trigeminal ganglion

\section{1 | Introduction}

Treatment of postherpetic neuralgia (PHN) is often only partially effective. If the lesion involves the trigeminal nerve, the treatment effect is often more unsatisfactory ${ }^{[1,2]}$. We describe here an elderly case of trigeminal postherpetic neuralgia which successfully treated by low-temperature plasma technology combined with lateral injection of trigeminal ganglion. 


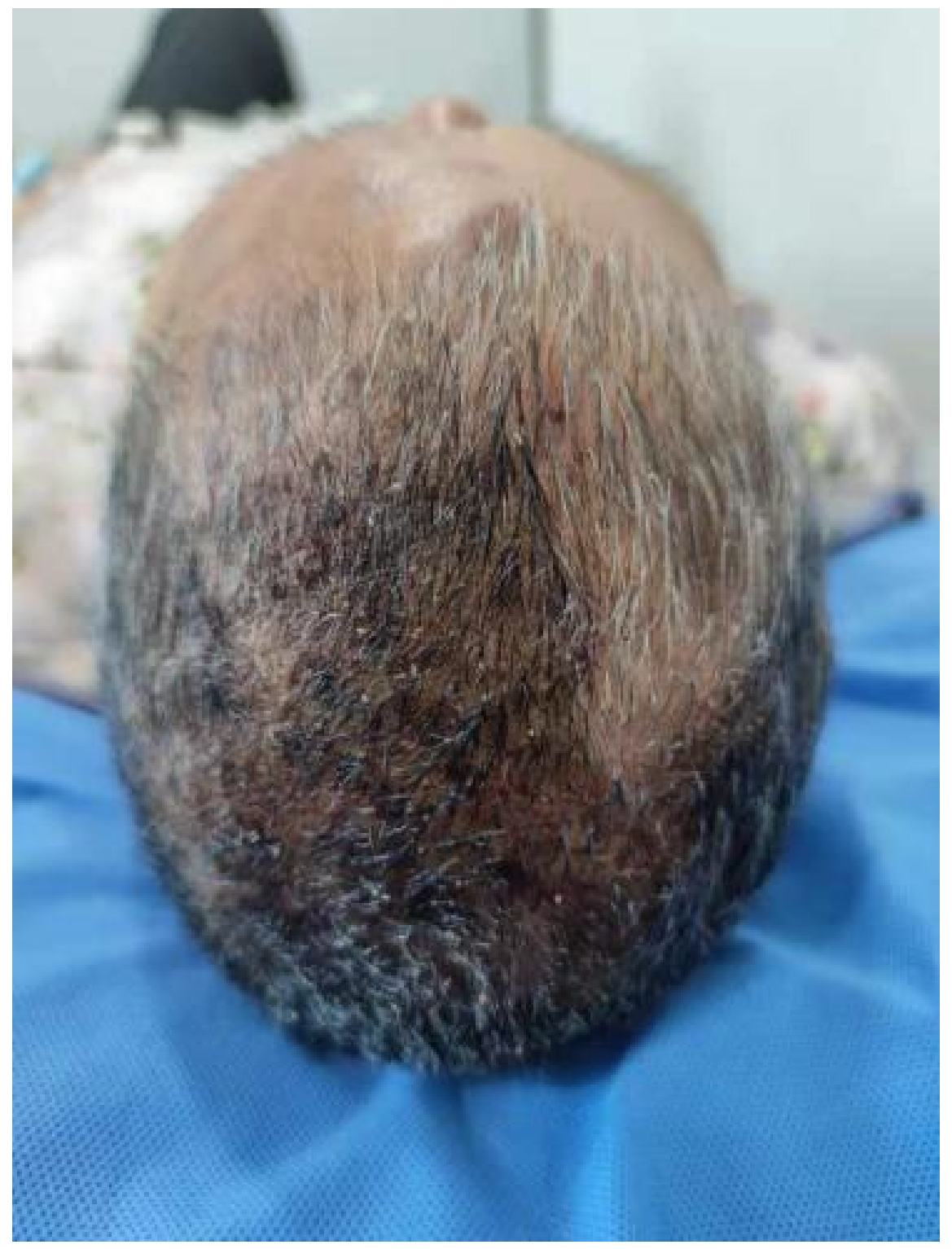




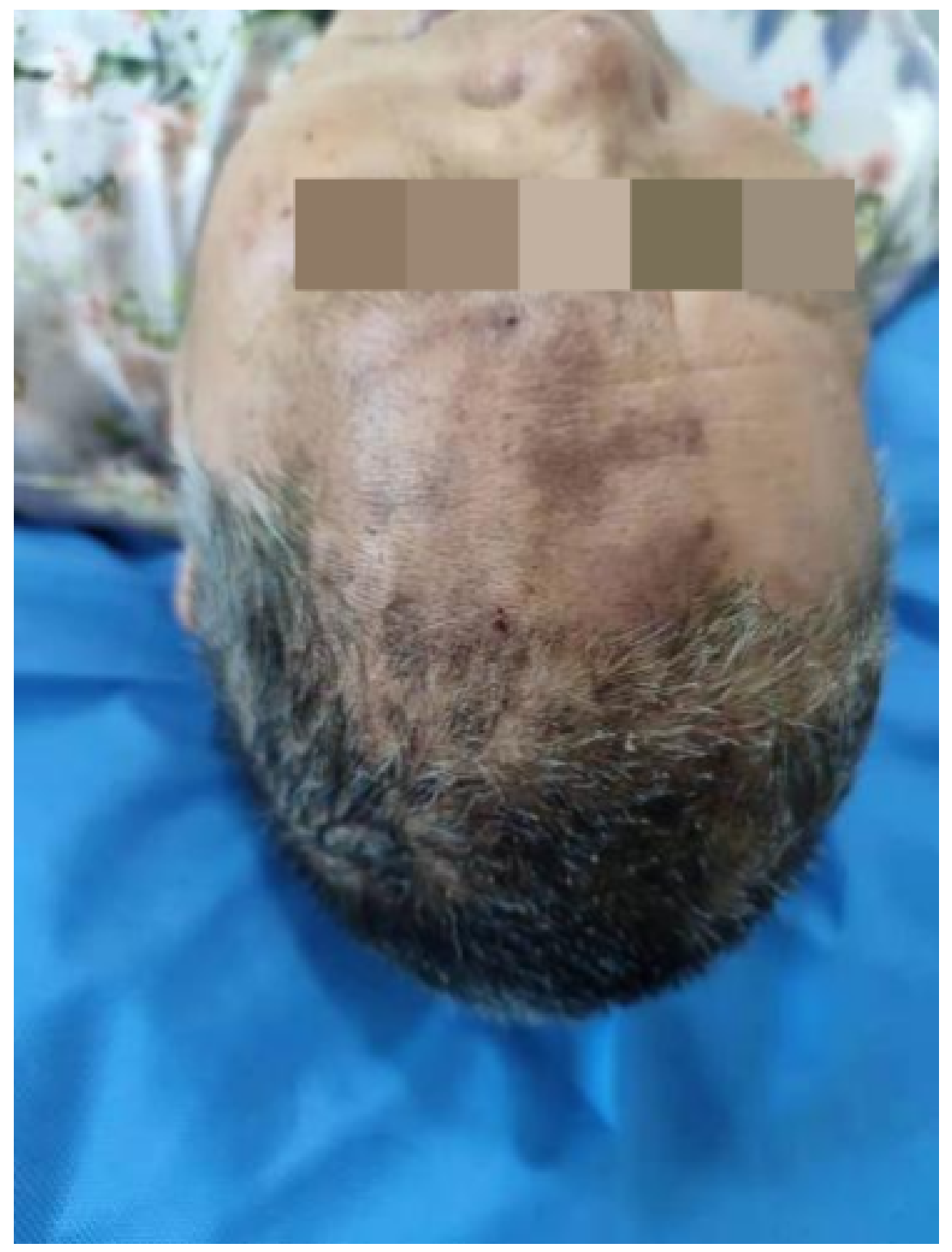




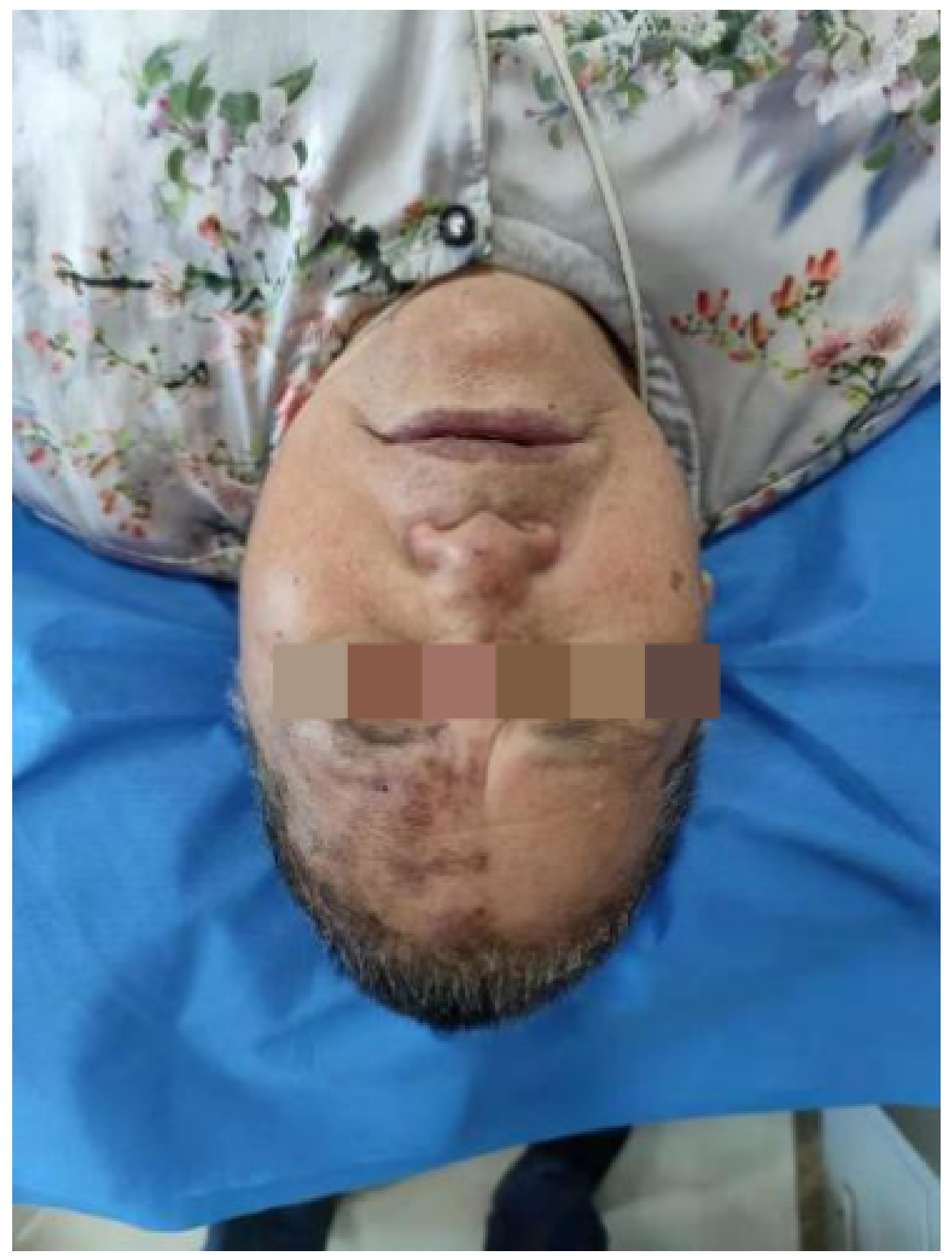

Fig 1.The patient has reddish-brown patchy pigmentation on the left upper eyelid, forehead, and crown, with scattered blisters on the skin of the crown. Blurred vision and photophobia in her left eye. VAS score:8-9.

\section{2 | CASE PRESENTATION}

The patient was a female, 74 years old. More than 20 days ago, there was no obvious inducement,but there was red herpes on the left forehead and top. It was diagnosed as "herpes zoster" in the local hospital. Antiviral, nerve nourishing and analgesic drugs were given intravenously to her. During the course of the disease, blurred vision and photophobia gradually appeared in the left eye. One week later, the herpes subsided, but the left forehead and orbit were still painful, with unclear vision and photophobia in the left eye (Fig 1.). He was hospitalized in our department and diagnosed as "postherpetic neuralgia". At admission, the patient complained of paroxysmal pain in the left frontal top and orbit, which was electric shock like. VAS score: 8-9 points, about 5 minutes each time, with an interval of 1-2 hours. SF-36 score ${ }^{[3-5]}$ : physiological function $(\mathrm{PF})$ : 65, physiological function (RF): 0, Body pain (BP): 22, general health status 
(GH): 57, energy (VT): 30, social function (SF): 22.22, emotional function (RE): 0, mental health (MH): 44, health change (HT): 25; Pittsburgh sleep quality index scale ${ }^{[6,7]}: 19$. Patchy-like dark red pigmentation can be seen in the distribution area of the first branch of the left trigeminal nerve, local tactility is normal without obvious tenderness, photophobia in the left eye, visual acuity is 0.2 , no obvious abnormality in the right eye, visual acuity is 0.5 . After admission, they were given ketochromate tromethamine $30 \mathrm{mg}$ bid, esmeprazole 40mg bid, acyclovir 0.25g tid, Mecobalamin $500 \mathrm{ug}$ qd intravenously, and vaccinated with cowpox vaccine, rabbit inflammatory skin extract $6 \mathrm{ml} \mathrm{qd}$; Oral pregabalin $75 \mathrm{mg}$ bid had poor pain control, and there was no significant change in attack and pain degree. With the consent of the patient, low temperature plasma nerve conditioning in the distribution area of the first branch of the trigeminal nerve was performed (Fig 2.). After entering the operating room, the patients were routinely opened venous access and ECG monitoring. The appropriate surgical position was selected according to the pain site, the pain site was fully exposed, and the local routine disinfection and towel laying were carried out. $20 \mathrm{ml}$ of $2 \%$ lidocaine $+20 \mathrm{ml}$ of $0.9 \%$ normal saline were prepared into $1 \%$ local anesthetic (without adrenaline) for subcutaneous local infiltration anesthesia. After the pain of local infiltration anesthesia disappears, enter the needle with the No. 12 puncture needle inclined $15^{\circ}$ with the skin. After reaching the subcutaneous area, take out the puncture needle core, insert the plasma knife head, connect the knife head with the plasma RF machine, adjust the ablation intensity of the RF machine at level 3, and the ablation time at each point is electric cutting for $5 \mathrm{~S}$ and electric coagulation for $5 \mathrm{~S}$. At the same time, swing the needle tail left and right to increase the ablation range. Every $0.5 \mathrm{~cm}$ is an ablation point, and ablation is performed while withdrawing the needle until all the painful parts are ablated. Due to the large pain area of the patient, the first operation is mainly in the most obvious areas of supraorbital foramen, subcutaneous prefrontal and top pain on the affected side. The next day, the operation is performed again, mainly in the most obvious areas above the hairline to the top pain. The operation site was kept clean and dry for $48 \mathrm{~h}$. Two operations completely covered the pain area of the patient.

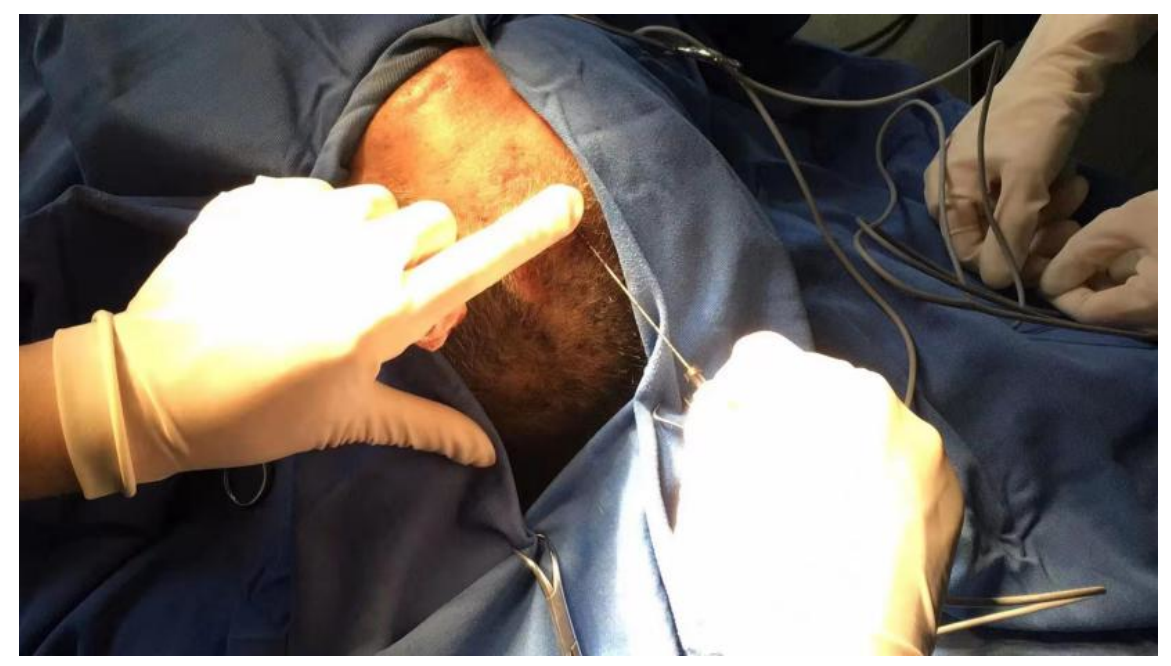

Fig 2. The surgical scope included the supraorbital hole and painful parts of the forehead and the top of the head. After local anesthesia with $1 \%$ lidocaine, the puncture needle was inserted into the skin. Every $0.5 \mathrm{~cm}$ was an ablation point, and the ablation time at each point is electric cutting for $5 \mathrm{~S}$ and electric coagulation for $5 \mathrm{~S}$.

After the initial operation, the interval between the onset of pain was prolonged to 3-4 hours, the duration was reduced to 1.5-2 minutes, and the forehead pain disappeared at the onset; After the second operation, the interval of pain attack was further extended to 5-6 hours, the duration was still 1.5-2 minutes, and the pain range was reduced to about $4.5^{*} 5 \mathrm{~cm}$ at the top.The trigeminal ganglion lateral approach was blocked once on the second and third day after operation. During the treatment, the patient took the supine position 
and turned his head to the healthy side. The lateral approach of trigeminal nerve semilunar ganglion through temporomandibular point was used. Disinfect the skin, spread sterile towel, and ask the patient to open the mouth slightly. The puncture point was the upper edge of the mandibular notch about $1 \mathrm{~cm}$ from the midpoint of the lower edge of the temporal arch to the tail. For local anesthesia with $1 \%$ lidocaine, uses a $5 \mathrm{ml}$ syringe needle from the puncture point close to the upper edge of the mandibular notch to pierce in the direction of $15^{\circ}-20^{\circ}$ backward and $15^{\circ}-30^{\circ}$ upward. The needle can reach about $4.5 \mathrm{~cm}$, and radiation pain can occur near the foramen ovale. Then withdraw the needle slightly and inject drugs (ketochromate aminobutanol, ribavirin injection, $2 \%$ lidocaine, $0.9 \%$ normal saline mixture) $2 \mathrm{ml}$. On the morning of the fourth day after operation, the patient complained that the pain of the previous night had not occurred, and the ocular photophobia symptoms were significantly improved. There was no recurrence of pain after 3 days in hospital, VAS score: 0 . After 30 days of follow-up, no pain occurred, VAS score: 0, SF-36 score: physiological function (PF): 70, physiological function (RF): 100, Body pain (BP): 72, general health status (GH): 67, energy (VT): 70, social function (SF): 100, emotional function (RE): 100, mental health (MH): 76, health change (HT): 50; Pittsburgh sleep quality index: 7 .

\section{3 | Discussion}

Trigeminal nerve is the main sensory nerve in the face. After the initial infection of herpes zoster, the virus is latent in the trigeminal ganglion. With the decline of human immunity, the latent herpes zoster virus can be reactivated and replicated, and the peripheral nerves associated with the infected ganglion will have lesions ${ }^{[8]}$. Severe inflammatory reactions can occur in the invaded ganglia, manifested as neuronal edema, necrosis and intraneural hemorrhage, resulting in neuropathic pain ${ }^{[9]}$.In addition, the peripheral sensitization mechanism is also considered to be one of the possible causes of PHN, which is mainly manifested in the ectopic discharge of damaged peripheral afferent fibers. Studies ${ }^{[10]}$ have shown that demyelinating changes and abnormal regeneration can occur after peripheral nerve injury, resulting in the formation of neuroma. Under the condition of no stimulation, neuroma can discharge spontaneously, while under chemical or mechanical stimulation, it will increase the discharge frequency of neuroma and cause severe pain. In the course of PHN, abnormal sprouting of small peripheral nerve fibers is similar to the external ectopic discharge in neuroma, which will cause abnormal feelings such as local skin pain and itching. Studies have shown that local anesthetics or proximal nerve block infiltrating painful skin can temporarily inhibit ongoing pain ${ }^{[11-13]}$. This shows that part of the pain caused by PHN comes from the pathological changes of peripheral fibers. At the same time, inhibiting the activity of skin ectopic discharge (pacemaker) can effectively alleviate the pain. In addition to the above theory of pain caused by nerve injury, some scholars believe that herpes zoster virus (VZV) still exists in the body at PHN stage and may cause pain symptoms.

Low temperature plasma ablation is a kind of minimally invasive interventional therapy. It is a new technology based on electrochemistry developed in recent years. It uses bipolar RF to generate energy to convert the electrolyte between the tissue and the RF cutter head into a plasma thin layer. The charged particles in the plasma are accelerated by the electric field to transfer energy to the tissue, dissociate the molecular bonds constituting cell components in the target tissue, and decompose the tissue into small molecular gases to achieve the purpose of tissue volume reduction. At present, low-temperature plasma technology is mostly used in the treatment of cervical and lumbar discogenic diseases. It is the first in our department to apply it to the treatment of neuropathic pain after herpes zoster.

Since 2016, more than 30 patients with intractable herpes zoster neuralgia have been treated. Statistics show that the effective rate is $86.67 \%$, and the excellent rate is $76.67 \%$. The main theoretical basis is: the temperature of the low-temperature plasma knife head is maintained at 40-70 ${ }^{[14]}$, while the nerve fibers conducting pain will denature only after 120 seconds above 70 . Therefore, this treatment will not cause the denaturation of normal nerve tissue, but it is possible to inactivate the abnormally sprouted unmyelinated terminal fibers, so as to correct the discharge and activity of ectopic pacing points of the skin, improving the internal environment of peripheral nerve, and finally achieve continuous remission of symptoms for a long time.Secondly, when the plasma cutter head works, it can instantly produce a large amount of exogenous reactive oxygen species (ROS) and reactive nitrogen (RNs). Some studies have shown that ROS and RNS 
play an important role in the potential biological effects of plasma ${ }^{[15-17]}$. ROS is involved in the transmission of cellular signals and is related to many biological systems ${ }^{[18]}$. It recruits many different mitogen activated protein kinases (MAPKs), resulting in the activation of cellular signal cascade ${ }^{[19]}$. This is likely to be involved in the mechanism of PHN pain regulation. In addition a large number of literatures have confirmed that low-temperature plasma has inactivation effect on microorganisms such as bacteria and viruses. Some theories believe that the occurrence of PHN may be related to VZV remaining in nerve tissue, so the treatment of PHN with low-temperature plasma technology can not only target the pathological changes of nerve tissue itself, but also inactivate the pathogenic microorganism VZV virus, which not only alleviates the existing pain of patients, but also fundamentally eliminates the possibility of recurrence or aggravation of pain.

After receiving low-temperature plasma nerve conditioning, the pain in some areas of the top was still not relieved. Combined with the current understanding of the mechanism of PHN, it is considered that the trigeminal ganglion may have inflammatory and hemorrhagic changes due to VZV. Therefore, puncture and injection through the lateral approach of trigeminal ganglion and local infiltration of antiviral, antiinflammatory and local anesthetic drugs were used in order to alleviate the inflammatory lesions of trigeminal ganglion and give targeted treatment to the possible VZV virus. The response after treatment was indeed as we expected, and the patient's symptoms disappeared. In this case, the first branch of the trigeminal nerve was involved. In addition to the pain symptoms, there were corneal involvement symptoms at the same time. After receiving low-temperature plasma nerve conditioning, not only the pain range, attack frequency and attack duration were significantly improved, but also the ocular symptoms were significantly relieved. There was no obvious abnormal sensation at the treatment site, and there were no other adverse sequelae. It can be seen that this method can not only treat the pain symptoms caused by PHN, but also protect the eye function for patients with the first branch of trigeminal nerve involved.

\section{ACKNOWLEDGMENTS}

Sun Yanjun is the guarantor of this work and, as such, had full access to all the data in the study and takes responsibility for the integrity of the data and the accuracy of the data analysis.

\section{CONFLICT OF INTEREST}

No potential conflicts of interest relevant to this article were reported.

\section{AUTHOR CONTRIBUTIONS}

Zhou Hao: was involved in the care and management of the patient, and all authors took part in the writing and reviewing of the article. Written consent for publication was obtained

from the patient.

\section{ETHICAL APPROVAL}

The patient gave informed consent to this publication.

\section{DATA AVAILABILITY STATEMENT}

Data are available in the patient's medical record.

\section{REFERENCES}

1. Gimenez-Mila M, Busquets C, Ojeda A, et al. Neuropathic pain with features of complex regional syndrome in the upper extremity after herpes zoster. Pain Pract 2014;14:158-61.

2. Alvarez FK, De Siqueira SR, Okada M, et al. Evaluation of the sensation in patients with trigeminal post-herpetic neuralgia. J Oral Pathol Med 2007;36:347-50.

3. Stewart AL,Hays RD, Ware JE Jr.The MOS short - form general health survey.Reliability and validity in a patient population.Medical Care,1988;26(7):724-735.

4. Eremenco SL,Celia D,Arnold BJ.A comprehensive method for the translation and cross - cultural validation of health status questionnaires.Evaluation\&the Health Professions.2005;28(2):212 - 232 . 
5. Terwee CB,Roorda LD,de Vet HC,et a1.Dutch-Flemish translation of 17 item banks from the patient-reported outcomes measurement information system(PROMIS).Quality of Life Research,2014;23(6):1733-1741.

6. Buysse DJ,Reynolds C3,Monk TH,et al.The Pittsburgh Sleep Quality Index:a new instrument for psychiatric practice and research.Psychiatry Res,1989;28(2):193-213.

7. Nierenbery AA,Adler LA,Peselow E,et al.Trazodone for antidepressant associated insomnia.Am J Psychia- try,1994;15l(7):1069-1072

8. Stinson C, Deng M, Yee MB, et al. Sex differences underlying orofacial varicella zoster associated pain in rats. BMC Neurol, 2017;17(1):95.

9. Steain M, Sutherland JP, Rodriguez M, et al. Analysis of T cell responses during active varicella-zoster virus reactivation in human ganglia. J Virol, 2014; 88(5):2704-2716.

10. WIESELER-FRANK J,MAIER S F,WATKINS L R. Immune- to-brain communication dynamically modulates pain: physiological and pathological consequences. Brain Behav Immun,2005,19( 2) : 104111.

11. Nurmikko T, Wells C, Bowsher D (1991) Pain and allodynia in postherpetic neuralgia: role of somatic and sympathetic nervous systems. Acta Neurol Scand 84:146-152

12. Puri N (2011) Modified Jaipur block for the treatment of post-herpetic neuralgia. Int J Dermatol 50:1417-1420

13. Riopelle JM, Naraghi M, Grush KP (1984) Chronic neuralgia incidence following local anesthetic therapy for herpes zoster. Arch Dermatol 120:747-750

14. KASCH R,MENSEL B,SCHMIDT F,et al. Disc volume reduction with percutaneous nucleoplasty in an animal model. PLoS One,2012;7( 11) : e50211.

15. Kalghatgi, S.et al. Effects of non-thermal plasma on mammalian cells. PLoS One 2011;6: e16270 .

16. Ahn, H. J. et al. Atmospheric-pressure plasma jet induces apoptosis involving mitochondria via generation of free radicals. PLoS One 2011;6, e28154.

17. Ahn, H. J. et al. Targeting cancer cells with reactive oxygen and nitrogen species generated by atmospheric-pressure air plasma. PLoS One 2014;9, e86173.

18. Mittler, R. et al. ROS signaling: the new wave? Trends. Plant Sci. 2011;16, 300-309.

19. Finkel, T. Signal transduction by reactive oxygen species. J. Cell Biol. 2011;194, 7-15 . 